\title{
High-density marker profiling confirms ancestral genomes of Avena species and identifies D-genome chromosomes of hexaploid oat
}

\author{
Honghai Yan ${ }^{1,2}$ - Wubishet A. Bekele ${ }^{1}$ Charlene P. Wight ${ }^{1} \cdot$ Yuanying Peng $^{2}$ • \\ Tim Langdon ${ }^{3} \cdot$ Robert G. Latta $^{4} \cdot$ Yong-Bi Fu ${ }^{5} \cdot$ Axel Diederichsen ${ }^{5}$. \\ Catherine J. Howarth ${ }^{3} \cdot$ Eric N . Jellen $^{6} \cdot$ Brian Boyle $^{7} \cdot$ Yuming Wei $^{2}$. \\ Nicholas A. Tinker ${ }^{1}$
}

Received: 17 June 2016 / Accepted: 2 August 2016 / Published online: 13 August 2016

(C) The Author(s) 2016. This article is published with open access at Springerlink.com

\begin{abstract}
Key message Genome analysis of 27 oat species identifies ancestral groups, delineates the $D$ genome, and identifies ancestral origin of 21 mapped chromosomes in hexaploid oat.

Abstract We investigated genomic relationships among 27 species of the genus Avena using high-density genetic markers revealed by genotyping-by-sequencing (GBS). Two methods of GBS analysis were used: one based on tag-level haplotypes that were previously mapped in cultivated hexaploid oat (A. sativa), and one intended to sample and enumerate tag-level haplotypes originating from all species under investigation. Qualitatively, both methods gave similar predictions regarding the clustering of species and shared ancestral genomes. Furthermore, results were consistent with previous phylogenies of the genus obtained with conventional approaches, supporting the robustness of whole genome GBS analysis. Evidence is presented to justify the final and definitive classification of the tetraploids
\end{abstract}

Communicated by M. E. Sorrells.

Electronic supplementary material The online version of this article (doi:10.1007/s00122-016-2762-7) contains supplementary material, which is available to authorized users.

Nicholas A. Tinker

nick.tinker@canada.ca

1 Ottawa Research and Development Centre, Agriculture and Agri-Food Canada, 960 Carling Ave., Ottawa, ON K1A 0C6, Canada

2 Triticeae Research Institute, Sichuan Agricultural University, Chengdu 611130, China

3 Institute of Biological, Environmental and Rural Sciences, Aberystwyth University, Gogerddan, Aberystwyth, Ceredigion SY23 3EE, UK
A. insularis, A. maroccana (=A. magna), and A. murphyi as containing D-plus-C genomes, and not A-plus-C genomes, as is most often specified in past literature. Through electronic painting of the 21 chromosome representations in the hexaploid oat consensus map, we show how the relative frequency of matches between mapped hexaploid-derived haplotypes and AC (DC)-genome tetraploids vs. A- and C-genome diploids can accurately reveal the genome origin of all hexaploid chromosomes, including the approximate positions of inter-genome translocations. Evidence is provided that supports the continued classification of a diverged $\mathrm{B}$ genome in $\mathrm{AB}$ tetraploids, and it is confirmed that no extant A-genome diploids, including $A$. canariensis, are similar enough to the $\mathrm{D}$ genome of tetraploid and hexaploid oat to warrant consideration as a D-genome diploid.

\section{Introduction}

Crop wild relatives are an important source of genetic variability for improving agricultural species through interspecific crossing and introgression (Zamir 2001). Cultivated oat has been improved through the interspecific transfer of alleles conferring resistance to disease (e.g., Aung et al.

4 Department of Biology, Dalhousie University, 1355 Oxford St., Halifax, NS B3H 4R2, Canada

5 Plant Gene Resources of Canada, Saskatoon Research and Development Centre, Agriculture and Agri-Food Canada, 107 Science Place, Saskatoon, SK S7N 0X2, Canada

6 Department of Plant and Wildlife Sciences, Brigham Young University, Provo, UT, USA

7 Plateforme d'analyses génomiques, Institut de biologie intégrative et des systèmes, Université Laval, Quebec City, QC G1V 0A6, Canada 
2010; Hoppe and Kummer 1991; Rines et al. 2007), and the transfer of other important quality and agronomic traits to cultivated oat has been initiated or proposed, as reviewed by Loskutov and Rines (2011). However, the use of crop wild relatives for introgression breeding is constrained by interspecific fertility barriers. Many interspecific hybrids are sterile, and those hybrids that can be made often require advanced techniques such as embryo rescue and bridging species, followed by extensive backcrossing, to achieve the transfer of a target allele (Loskutov 2001). Therefore, it is important to develop detailed information regarding the value and transferability of traits from crop wild relatives prior to investing resources into introgression breeding. The genus Avena shows a wide variation in genome size, from 4.1 to $12.8 \mathrm{Gbp}$, which is determined primarily by ploidy level, but may also be influenced by variation in the types and amounts of repeated DNA among genomes (Yan et al. 2016). Differences among genomes of cultivated hexaploid oat and its wild relatives will probably determine the locations into which foreign genes are introgressed, and may also influence the amount of linkage drag or the propensity for chromosomal rearrangements involving introgressed chromosomes. Thus, detailed chromosome-level knowledge of the structural and ancestral relationships among the genomes of Avena species is of both practical and theoretical interest.

The genus Avena contains up to 30 recognized species (see Table 1, which includes species authorities) in a series of ploidy levels that includes diploids, tetraploids, and hexaploids (Baum 1977; Baum and Fedak 1985a, b; Ladizinsky 1998). A number of the recognized species exhibit high degrees of cross fertility, contrary to the biological species definition (e.g. Ladizinsky 2012). The diploid species fall clearly into two major genome groups: A and C. All hexaploid species, including the cultivated oat, A. sativa, are described as having an ACD genome composition, corroborated by fertile interspecific crosses among the hexaploids, as well as by their near-identical genome sizes (Yan et al. 2016). Less clearly, the tetraploids have been assigned designations of $\mathrm{CC}, \mathrm{AA}$ or $\mathrm{AB}$, and $\mathrm{AC}$ or DC. Genomes designated as B and D within the polyploids have not been shown to exist within extant diploid species. Debate remains regarding which $\mathrm{A}$ - and $\mathrm{C}$-genome diploids are closest to the $\mathrm{A}$ and $\mathrm{C}$ genomes of hexaploid oat, and whether or not any extant A-genome diploid is closely related to the $\mathrm{B}$ and/or $\mathrm{D}$ genomes.

Two points of clarification should be noted regarding the genome designations in oat. First, these designations contain no deliberate implications of orthology to wheat or any other non-Avena genomes with the same alphabetical genome designations. Second, there are no established rules on how different two genomes need to be before they are designated by a different letter. So, while the A and C genomes of oat are clearly differentiated, the designations of B and D genomes and the naming of different A-genome sub-types are subject to debate. In this work, we take the viewpoint that these designations can be used for pragmatic purposes if they are informative of a degree of divergence that can be reliably and repeatedly measured.

Structural differences among the A-genome diploid species have been revealed by karyotype analysis (Fominaya et al. 1988; Rajhathy and Thomas 1974) and by the chromosome pairing of their hybrids (Leggett 1989; Thomas 1992), resulting in the division of the $A$ genome into subclassifications of $A_{s}, A_{c}, A_{d}, A_{l}$, and $A_{p}$ genomes (Table 1). Although no A-genome diploid has shown a perfect karyotype match to the hexaploid A genome, diploids with the $\mathrm{A}_{\mathrm{s}}$ genome sub-type have shown the highest degree of chromosome pairing in hybrids with hexaploid species (Kihara and Nishiyama 1932; Marshall and Myers 1961; Rajhathy and Morrison 1960). Furthermore, the $\mathrm{A}_{\mathrm{s}}$ genome species have also shown the closest karyotype matches (Rajhathy and Thomas 1974), in situ hybridization patterns (Chen and Armstrong 1994; Jellen et al. 1994; Linares et al. 1996, 1998) and molecular similarity (Peng et al. 2010; Yan et al. 2014) to the hexaploid species.

Since the D genome of hexaploid oat shows high similarity to the A genome, it has been suggested that the D genome may be a diverged version of the A genome from a recent diploid ancestor that has yet to be identified, or may no longer be extant (Loskutov 2008). Based on evidence from morphological features (Baum et al. 1973), isoenzyme variations (Craig et al. 1974), and fluorescent in situ hybridization (FISH) results (Linares et al. 1998), there is unpublished speculation that the $\mathrm{A}_{\mathrm{c}}$ genome variant found in A. canariensis might be the closest surviving diploid genome to the ancestral $\mathrm{D}$ genome donor.

The $\mathrm{C}$ genome of hexaploid oat is highly diverged from the $\mathrm{A}$ and $\mathrm{D}$ genomes, as demonstrated by substantially more pronounced C-banding (Fominaya et al. 1988; Jellen et al. 1993), and these differences have been substantiated by differential FISH analysis (Hayasaki et al. 2000; Jellen et al. 1994; Linares et al. 1998; Yang et al. 1999). There are three $\mathrm{C}$-genome diploid species, which have been further subclassified as having two genome types $\left(\mathrm{C}_{\mathrm{p}}\right.$ and $\mathrm{C}_{\mathrm{v}}$ ) because of minor structural differences (Rajhathy and Thomas 1967). Based on cytogenetic and molecular evidence, both types have been proposed as being contributors of the $\mathrm{C}$ genome in hexaploid species (Chen and Armstrong 1994; Nikoloudakis and Katsiotis 2008; Peng et al. 2010; Rajhathy 1966).

There are four species commonly considered to have $\mathrm{AB}$ genomes. Of these, A. barbata, A. vaviloviana, and A. abyssinica may belong to a common biological species, while A. agadiriana is distinct (Ladizinsky 2012). Based on FISH analysis using an A-genome satellite probe, Irigoyen et al. 
Table 1 List of Avena species included in this work, classification by genome and PCoA group, and proportion of mapped loci (hexaploid GBS model) present

\begin{tabular}{|c|c|c|c|c|c|c|}
\hline Species/group $^{\mathrm{a}}$ & Hap-lome $^{\mathrm{b}}$ & Ploidy $(2 n)$ & Mean $2 \mathrm{C}$ values $(\mathrm{pg} \text { DNA })^{\mathrm{c}}$ & Num. $^{\mathrm{d}}$ & Mapped loci $(\%)^{\mathrm{e}}$ & Haplotypes per shared locus ${ }^{\mathrm{f}}$ \\
\hline A. fatua $\mathrm{L}$. & ACD & $6 \times$ & 25.8 & 8 & 91.3 & 1.58 \\
\hline Avena $\times$ glabrata Hausskn. & ACD & $6 \times$ & NA & 1 & 81.0 & 1.17 \\
\hline Avena $\times$ haussknechtii Nevski & $\mathrm{ACD}$ & $6 \times$ & NA & 1 & 73.0 & 1.02 \\
\hline A. hybrida Peterm. & $\mathrm{ACD}$ & $6 \times$ & NA & 5 & 86.1 & 1.27 \\
\hline A. occidentalis Durieu & ACD & $6 \times$ & 25.7 & 6 & 90.6 & 1.45 \\
\hline A. sativa $\mathrm{L}$. & $\mathrm{ACD}$ & $6 \times$ & 25.7 & 119 & 99.0 & 1.91 \\
\hline A. sterilis $\mathrm{L}$. & $\mathrm{ACD}$ & $6 \times$ & 25.8 & 17 & 94.5 & 1.69 \\
\hline Group 1 & ACD & $6 x$ & 25.8 & 157 & 99.7 & 1.96 \\
\hline A. insularis Ladiz. & $\mathrm{AC}(\mathrm{DC})$ & $4 \times$ & 18.6 & 3 & 58.6 & 1.14 \\
\hline A. maroccana Gand. & $\mathrm{AC}(\mathrm{DC})$ & $4 \times$ & 18.5 & 4 & 49.0 & 1.06 \\
\hline A. murphyi Ladiz. & $\mathrm{AC}(\mathrm{DC})$ & $4 \times$ & 18.7 & 1 & 34.2 & 1.00 \\
\hline Group 2 & $\mathrm{AC}(\mathrm{DC})$ & $4 x$ & 18.6 & 8 & 66.7 & 1.25 \\
\hline A. atlantica Baum et Fedak & $\mathrm{A}_{\mathrm{s}}$ & $2 \times$ & 9.2 & 3 & 10.3 & 1.05 \\
\hline A. brevis Roth & $\mathrm{A}_{\mathrm{s}}$ & $2 \times$ & 9.0 & 6 & 10.6 & 1.04 \\
\hline A. hirtula Lag. & $\mathrm{A}_{\mathrm{s}}$ & $2 \times$ & 9.1 & 2 & 11.1 & 1.04 \\
\hline A. hispanica Ard. & $\mathrm{A}_{\mathrm{s}}$ & $2 \times$ & 8.8 & 6 & 10.7 & 1.04 \\
\hline A. nuda $\mathrm{L}$. & $A_{s}$ & $2 \times$ & 9.1 & 6 & 9.6 & 1.01 \\
\hline A. strigosa Schreb. & $\mathrm{A}_{\mathrm{s}}$ & $2 \times$ & 9.1 & 16 & 14.2 & 1.09 \\
\hline A. wiestii Steud. & $\mathrm{A}_{\mathrm{s}}$ & $2 \times$ & 9.1 & 2 & 11.2 & 1.05 \\
\hline Group 3 & $\mathbf{A}_{\mathrm{s}}$ & $2 x$ & 9.1 & 41 & 22.3 & 1.08 \\
\hline $\begin{array}{l}\text { A. longiglumis Durieu } \\
\text { (=Group 4) }\end{array}$ & Al & $2 x$ & 9.2 & 3 & 10.4 & 1.05 \\
\hline A. canariensis Baum et al. & $\mathrm{A}_{\mathrm{c}}$ & $2 \times$ & 8.8 & 5 & 5.6 & 1.01 \\
\hline $\begin{array}{l}\text { A. damascena Rajhathy } \\
\text { et Baum }\end{array}$ & $A_{d}$ & $2 \times$ & 8.4 & 3 & 6.0 & 1.01 \\
\hline A. lusitanica Baum & $\mathrm{A}_{\mathrm{s}}$ & $2 \times$ & 8.7 & 2 & 7.2 & 1.02 \\
\hline Group 5 & $\mathbf{A}_{\mathrm{c} / \mathrm{d}}$ & $2 x$ & $\mathbf{V}$ & 10 & $\mathbf{V}$ & 1.05 \\
\hline A. abyssinica Hochst. & $\mathrm{AB}$ & $4 \times$ & 16.7 & 6 & 12.2 & 1.04 \\
\hline A. barbata Pott ex Link & $\mathrm{AB}$ & $4 \times$ & 16.4 & 5 & 14.6 & 1.10 \\
\hline A. vaviloviana Mordv. & $\mathrm{AB}$ & $4 \times$ & 16.4 & 4 & 12.0 & 1.04 \\
\hline Group 6 & $\mathbf{A B}$ & $4 x$ & 16.5 & 15 & 15.7 & 1.10 \\
\hline $\begin{array}{l}\text { A. agadiriana Baum et Fedak } \\
\text { (=Group 7) }\end{array}$ & $\mathbf{V}$ & $\mathbf{V}$ & 17.5 & 4 & 10.7 & 1.06 \\
\hline A. clauda Durieu & $\mathrm{C}_{\mathrm{p}}$ & $2 \times$ & 10.3 & 3 & 4.2 & 1.01 \\
\hline A. eriantha Durieu & $\mathrm{C}_{\mathrm{p}}$ & $2 \times$ & 10.2 & 3 & 5.1 & 1.02 \\
\hline A. ventricosa Balansa ex Coss. & $\mathrm{C}_{\mathrm{v}}$ & $2 \times$ & 10.3 & 2 & 4.5 & 1.00 \\
\hline Group 8 & $\mathbf{C}$ & $2 \times$ & 10.3 & 8 & 5.6 & 1.02 \\
\hline $\begin{array}{l}\text { A. macrostachya Balansa et } \\
\text { Durieu (=Group 9) }\end{array}$ & $\mathrm{C}_{\mathrm{m}} \mathrm{C}_{\mathrm{m}}$ & $4 x$ & 21.8 & 1 & 2.1 & 1.02 \\
\hline
\end{tabular}

${ }^{a}$ Groups and single species that form groups (bold) are based on the established genome subgroup supported by PCoA analysis (Fig. 1). Species that are considered in this work to be homotypic include: A. nudi-brevis $(=A$. nuda), A. pilosa (=A. eriantha), A. ludoviciana (=A. sterilis), A. byzantina (=A. sativa), A. prostrata (=A. hirtula) and A. Magna (=A. maroccana). Avena $\times$ glabrata is a hybrid of A. sativa and A. fatua, while Avena $\times$ haussknechtii Nevski is hybrid of A. sativa and A. sterilis, according to Baum (1977). The incorrect use of "A. nuda" to refer to a hulless hexaploid species has been corrected to the accepted classification within A. sativa. References for authorities are provided by Loskutov and Rines (2011)

b Most commonly referenced genome constitution, with alternate speculations in parentheses

${ }^{c}$ Mean genome size reported by Yan et al. (2016). For groups, these values are reported as the unweighted arithmetic mean of species included in the group

d Number of accessions after removal of outliers (see Online Resource 1)

e Percentage of the total number of loci in the hexaploid GBS model where one or more of the hexaploid haplotypes were found in the respective species or ancestral genome group

${ }^{\mathrm{f}}$ Average number of hexaploid haplotypes found at loci where at least one haplotype was found in the respective species or ancestral group 
(2001) found a clear distinction between A- and B-genome chromosomes within both A. barbata and A. vaviloviana, justifying their designation as allotetraploids. This was supported by the identification of two different haplotypes of nuclear Acc1 genes from AB-genome tetraploids (Yan et al. 2014). In contrast, Chew et al. (2016) found that ABgenome tetraploids clustered closely with A-genome diploids; thus, they suggested that the B genome designation is not justified. Most work, including that of, Chew et al. (2016) has supported a distinction between A. agadiriana and other so-called AB-genome tetraploids. A. agadiriana differs karyotypically from other $\mathrm{AB}$ genome tetraploid species (Badaeva et al. 2010; Jellen and Gill 1996) and molecular analysis has revealed substantial differences between A. agadiriana and the barbata group (Peng et al. 2010; Yan et al. 2014). This may indicate that $A$. agadiriana contains a different combination of $\mathrm{A}$ and/or $\mathrm{B}$ genomes from the A. barbata group. Since A. agadiriana shares similarities with the tetraploids in the AC (DC) genome group (Alicchio et al. 1995), as well as with some hexaploid oats (Badaeva et al. 2010), there is also speculation that this species may contain an $\mathrm{AD}$-genome combination.

It has been proposed that hexaploid oat originated through the formation of an AC genome tetraploid from A and $\mathrm{C}$ genome diploids, followed by hybridization to a $\mathrm{D}$ genome diploid, with chromosome doubling at each stage to stabilize chromosome pairing (Loskutov 2008; Thomas 1992). This proposal is based partially on the existence of three tetraploid species, A. insularis, A. maroccana, and A. murphyi, which are currently designated as having $\mathrm{AC}$ genomes. The AC designation is based on genomic in situ hybridization (Leggett et al. 1994), as well as on the close genetic and morphological proximity of these tetraploids to the hexaploids (Ladizinsky 1971, 1998; Li et al. 2000; Murphy et al. 1968). However, these AC genome designations have been challenged by new molecular evidence (Oliver et al. 2011a, b; Peng et al. 2008; Peng et al. 2010; Yan et al. 2014), which, together with previous observations of meiotic chromosome pairing (Ladizinsky 1969) and FISH analysis (Linares et al. 1998), suggests that these tetraploids may contain the D genome found in hexaploid oat. The validation of the existence of the $\mathrm{D}$ genome in tetraploid oat, and a clear molecular delineation between chromosomes of A vs. D origin, would lead to a better understanding and utilization of oat genetic resources.

Many of the above inferences regarding genome evolution in Avena have been made using alignments of conserved nuclear or chloroplast genes. Such techniques are favoured because they allow concise estimates of the number and order of genetic mutations within and between genomes (using nuclear genes) or interferences about maternal parents (using chloroplast genes). However, single genes may not represent the average rate of divergence across entire genomes, and may not show adequate diversity to resolve differences within a species or between closely-related species. When reference genomes are available, genome resequencing can be used to construct phylogenies based on many gene sequences. Alternatively, alignments can be built using transcriptomes or captured exomes. However, like many other plants with large duplicated genomes, genome reference sequences are not yet available for species of Avena, and large-scale alignments are confounded by the presence of homeologues. As an alternative to these methods, inferences of genetic divergence can be made using composite statistics from molecular marker analysis. Several previous studies of Avena using various types of molecular markers have shown a general consensus in the qualitative clustering of species that agrees with most single-gene analyses (Alicchio et al. 1995; Chew et al. 2016; Drossou et al. 2004; Fu and Williams 2008; Li et al. 2000; Nocelli et al. 1999). Of these, only Fu and Williams (2008) used adequate numbers of accessions to address genetic diversity within species, while the most recent study by Chew et al. (2016) is notable for the use of high-density genotyping-by-sequencing (GBS) markers. While molecular markers provide superior genome coverage to single-gene studies, marker assays may confound allelic polymorphism with genetic differences among paralogous loci. Thus, interspecific comparisons using anonymous genetic markers may give biased estimates of distance, particularly when comparing species with different ploidy levels. Furthermore, none of the above studies have used markers with well-characterized map positions that would enable chromosome-based inferences of genetic distance.

Recently, progress has been made in the development of a high-density consensus linkage map in A. sativa (Chaffin et al. 2016) based on high throughput array-based SNPs (Tinker et al. 2014) and GBS markers (Huang et al. 2014). This map not only improved substantially upon the previous consensus map (Oliver et al. 2013), but also identified uncertainties and ambiguities in the physical chromosome assignments that had been proposed. As a result, only nine of the 21 hexaploid chromosome representations in the current consensus map are assigned with certainty to physical chromosomes using the most recent chromosome nomenclature proposed by Sanz et al. (2010). However, Chaffin et al. (2016) also illustrated the possibility of assigning chromosomes to genomes based on the similarity of mapped marker sequences to partially sequenced ancestral genomes. We recognized that this concept could be extended through the use of high-density GBS profiling in additional Avena species, and that this strategy could help to elucidate a more detailed understanding of the ancestral genomes of hexaploid oat. In preparation for this work, we developed a novel GBS pipeline called 'Haplotag' (Tinker et al. 2016) which 
was designed to discover and assign tag-level haplotypes to discrete diploid loci in a systematic way using population-level filters. We reasoned that, by identifying tag-level haplotypes that contain multiple SNPs, we could achieve a more sensitive detection of ancestral alleles than was possible using single SNP analysis (Tinker et al. 2016).

The objectives of this current work were to examine representative accessions from all available and recognized species of Avena using GBS analysis, and to identify their similarities to hexaploid oat based on exact matches to taglevel haplotypes discovered in hexaploid oat. From this, we planned to validate or advance hypotheses about the evolutionary origins of hexaploid oat on a chromosome and mapbased level. Furthermore, we sought to explore the allelic diversity of hexaploid oat ancestors on a per-locus basis, leading to recommendations for germplasm conservation and utilization. Because of the above-mentioned limitations of genetic markers, it was not our intention to perform a definitive phylogenetic analysis of the genus Avena. Nevertheless, by analysing genetic similarities among a comprehensive set of Avena species that is supported by classical phylogenetic studies, we hoped to evaluate the generalized use of GBS in phylogeny analysis and to comment on its potential use in other species where previous phylogenies are lacking.

\section{Materials and methods}

\section{Plant material}

Seeds of 266 accessions representing 27 Avena species and two hexaploid oat hybrids were obtained from either Plant Gene Resources of Canada (PGRC) or the United States Department of Agriculture Germplasm Resources Information Network (Table 1 and Online Resource 1). To reduce the potential for misclassification and seed mixtures, at least three accessions per species were assayed when available. Furthermore, some accessions were replicated by sequencing multiple plants from within accessions. The species A. atherantha, A. matritensis, and A. trichophylla (Baum 1977) were not included because of a lack of viable material. Although there remains controversy regarding the distinctness and discovery of A. maroccana vs. A. magna (Ladizinsky 1994), both species names are used synonymously in the literature for the same accessions, and we have chosen the former. Other species that we considered to be homotypic are noted in Table 1 .

\section{DNA extraction, library construction, and sequencing}

Leaf tissue from single plants of each accession was collected in paper envelopes, each containing a $10 \mathrm{~g}$ indicating silica gel pouch (silicagelpackets.ca). These were then placed inside large, re-sealable plastic bags until the tissue was dry. Dried leaf tissue was ground in $2 \mathrm{ml}$ plastic tubes containing two $3.1 \mathrm{~mm}$ stainless steel beads (Fox Industries, Fairfield, NJ, USA) each using a Tissuelyser bead mill (Qiagen Inc., Mississauga, ON, Canada). DNA was isolated using DNeasy Plant Mini kits (Qiagen Inc., Mississauga, ON, Canada). Complexity reduced, multiplexed GBS libraries were constructed by the Plateforme d'Analyses Génomiques of the Institut de Biologie Intégrative et des Systèmes (IBIS), Université Laval, Quebec City, Canada, based on the PstI-MspI method described by Huang et al. (2014). Complexity reductions were multiplexed using barcode adapters, with 96 samples per pooled library. Sequencing of each pooled library was performed on a single lane of a HiSeq 2500 platform (Illumina, San Diego, CA, USA) using standard Illumina protocols and kits, producing high-output paired-end 150 bp reads. Although $100 \mathrm{bp}$ single-end sequencing was adequate to support the current methods, we chose to perform longer paired-end reads to support future work.

\section{Generating tag count files and tag-level haplotypes (hexaploid model)}

Raw sequence files in FASTQ format were processed using the first step in the UNEAK-GBS pipeline (Lu et al. 2013) to trim the reads, de-convolute the barcodes, and produce a single tag count file for each sample. The tag count files, along with a locus definition file and a haplotype definition file, were used as input for the program Haplotag (Tinker et al. 2016), which was run in 'production mode'. The latter two files were from previous work where Haplotag was used in 'cluster discovery mode' with a cumulative set of 4657 A. sativa accessions from multiple studies, intended to develop a consistent set of reference tags from cultivated oat. The full set of 353,133 tags and 164,741 locus assignments from these files are available in Online Resource 2. When Haplotag was run in 'production mode' using these input files, the GBS analysis was restricted to the enumeration of tag-level haplotypes that were previously discovered in A. sativa. The result was a matrix of samples by loci, with an alphabetical string of tag-level haplotypes assigned to each cell. This was then converted to a binary (plusminus) matrix, with ' 1 ' representing the presence of any tag-level haplotype at a given locus, and ' 0 ' representing the absence of any haplotype.

\section{Sample validation and PCoA of samples (hexaploid model)}

To ensure the accuracy of species and genome classifications, the identities of many accessions were validated by 
estimating genome size using flow cytometry (as described by Yan et al. 2016). Then, all samples were subjected to a principal coordinates analysis ( $\mathrm{PCoA})$ of the binary matrix described above based on the Dice dissimilarity index estimated using the package 'arules' in the program ' $R$ ' ( $R$ Core Team 2016). Samples with $2 C$ values that conflicted with the accepted ploidy level of the species, and samples that clustered differently from the majority of cohorts within a species, were excluded from further analysis, as noted in Online Resource 1. The PCoA analysis was then repeated to visualize proximity and grouping of samples from validated accessions.

\section{Merging and normalization of tag counts}

Tag count files of validated samples from the same species were merged by summing the counts for the union of all common tags. Following this, the species-level tag count files were down-sampled until all diploid species had the same total tag counts (the sum of tag counts across all tag types $=1 \times$ ), and tetraploid and hexaploid species had total counts of $2 \times$ and $3 \times$, respectively. Down-sampling was performed by generating a new whole-number count for each tag based on a down-sampled expectation. For example, to down-sample a total tag count from $3 \times 0^{6}$ to $2 \times 10^{6}$, a given tag that was observed 600 times would be down-sampled to exactly 400 , while a tag that was observed only once would have a $33.3 \%$ probability of becoming zero and a $66.6 \%$ probability of remaining as one. In one special case, the species A. macrostachya (having only one accession, thus the lowest number of tag counts) was upsampled using the same methods. Tag count files were also merged and normalized by ancestral groups, as validated by PCoA results, to provide the deepest possible GBS tag count for each ancestral group. These normalized tag count files were used in subsequent analyses, as noted.

\section{Genotype analysis from de novo haplotype discovery}

A second GBS analysis was performed based on de novo haplotype discovery using the normalized species-level tag counts. First, all tags that were observed between 20 and 50 times within a species were identified as 'key tags'. The lower limit (20) was chosen to favour tags that were represented efficiently in the sequenced GBS library, while the upper limit was set to avoid including tags from repetitive elements. To reduce ascertainment bias further, the number of key tags contributed by each species was truncated to that of the species with the smallest number of key tags. Following this, the remaining key tags were joined by union to form a set of key tags intended to represent all species. Next, a plus-minus matrix of key tags by species was tabulated from the normalized tag count files, this time counting any number of observed tags $(>0)$ within a species as present. These data were subjected to PCoA analysis using the Dice dissimilarity index, as described earlier.

\section{In silico polyploid analysis}

To infer potential polyploidization events from extant species, in silico hexaploids were created by merging normalized tag counts from selected diploid and tetraploid species. Since ploidy was already compensated for in the normalized tag count files (a diploid contributed $1 \times$ tags and a tetraploid contributed $2 \times$ ), the total number of tags in the derived hexaploids were all equal (at $3 \times$ ) and required no further normalization. All possible combinations of one A-genome diploid species plus one AC (DC) genome tetraploid species, as well as one $\mathrm{C}$-genome diploid species plus one $\mathrm{AB}$ genome tetraploid species, were generated. Genetic similarities among extant and in silico hexaploid species were estimated using the Dice dissimilarity index based on the plus-minus scores from the de novo GBS model, and a PCoA analysis was conducted as described above. Hierarchical clustering by the unweighted pair group method with arithmetic mean (UPMGA) was also used to explore the proximity of extant and in silico species. A similar strategy was employed to explore in silico tetraploids formed from extant diploids.

\section{Comparative chromosome E-painting by species and ancestral group}

In previous work, markers discovered in the Haplotag hexaploid model were placed on the hexaploid consensus map using the method described by Chaffin et al. (2016). This produced an expanded linkage map called 'Expanded Oat Consensus Map 2016' containing 50,668 loci, which is available for download from the public oat database 'T3/ Oat' (https://triticeaetoolbox.org/oat/). From this, a mapped subset of the loci and tag-level haplotypes from the complete hexaploid model was selected (Online Resource 2). These tags, along with the normalized tag counts from each species and each ancestral group (as defined by PCoA), were used in a Haplotag production model to generate a text-based matrix (genotypes represented by character strings) and a plus-minus matrix (presence-absence of hexaploid haplotypes recorded as ' 1 ' or ' 0 '). The overall density of shared hexaploid tag-level haplotypes for each species and ancestral group was calculated as the frequency of 'plus' values in the plus-minus scoring matrix. A haplotype diversity index was also computed by counting all characters in the text-based scoring matrix and dividing by the number of 'plus' values in the plus-minus matrix. The scoring matrices were then smoothed and scaled using a sliding $30 \mathrm{cM}$ window to summarize the chromosome 
region surrounding each $1 \mathrm{cM}$ increment of the consensus map. The frequency of loci with 'plus' scores (locus-presence) was recorded within each window, and the average number of haplotypes per 'plus' locus (haplotype-diversity) was calculated.

The smoothed locus-presence and haplotype-diversity profiles were then interpreted using electronic painting (E-painting) on circle graphs of the oat consensus map to show where each species or ancestral group exceeded a given threshold. These thresholds were intended to delineate the relative frequency within a given species or group rather than the absolute frequencies. Since an idealized diploid would contain $1 / 3$ of the hexaploid haplotypes and an AC (DC) tetraploid would contain $2 / 3$, we set these thresholds to show where locus presence or haplotype diversity exceeded the 33rd or 66th percentiles within each species or group, respectively. For the haplotype-diversity profiles, these thresholds were adjusted slightly to achieve better differentiation among chromosomes. Circle diagrams were created using 'Circos' software Version 6.7 (Krzywinski et al. 2009).

\section{Results}

\section{Raw data and GBS tags}

Raw data and tag sequences from the GBS analyses are recorded in Online Resource 2, as described below and in the first index sheet of the online resource. All of the 353,133 tags assigned to 164,741 hexaploid loci (Sheet 1) were used to generate a presence-absence matrix of loci-by samples (Sheet 2). Of these, 26,910 loci placed on the hexaploid oat consensus map (Sheet 3 ) were used in the map-based chromosome E-painting. In the de novo analysis, a total of 85,831 unique tag-level haplotypes (Sheet 4 ) were scored across species and in silico polyploids (Sheet 5).

\section{Validation of accessions}

Initially, a set of 672 samples representing 266 accessions was examined using PCoA analysis. Based on this result, as well as on flow cytometry and phenotypic observations (not shown), 40 samples were considered to be misclassified and were removed, leaving 632 samples from 247 accessions for further analysis. A list of the samples that were removed is shown in Online Resource 1, since this information may assist with future genebank curations. For each of these accessions, we provide a possible reclassification by species and/or genome group; however, caution should be applied in using this information. In some cases, where we tested multiple seeds from an accession, we observed heterogeneous results. So, although these misclassifications represent a small proportion of accessions compared with those considered to be correct, the heterogeneity of species classifications that we observed in some accessions could potentially exist within a few correctly classified accessions where we tested only one sample. A deeper analysis of within-accession heterogeneity will be the subject of a forthcoming study.

\section{Genomic relationships among Avena species}

Relationships among Avena species were visualized using PCoA analysis on individual samples based on mapped loci (Fig. 1) as well as on a per species basis using de novo haplotypes (Online Resource 3). Both analyses revealed similar groupings by species, supporting a consistent nomenclature of nine different ancestral groups (Fig. 1; Table 1; and Online Resource 3). Because the map-based GBS analysis was conducted on a per-sample level, and because it provided the clearest separation among species and ancestral groups, we will describe those results, drawing attention to the de novo analysis only where results were less consistent.

All of the hexaploids formed a homogeneous group (Group 1) that was separated from other species. Three tetraploid species, A. insularis, A. maroccana, and A. murphyi clustered together into Group 2, and showed the highest similarity $(66.7 \%)$ to hexaploid oats based on shared haplotypes (Table 1). Within this group, A. insularis showed a higher frequency $(58.6 \%)$ of shared hexaploid haplotypes than A. maroccana (49.0\%) or A. murphyi (34.2), and appeared to cluster most closely with the hexaploid accessions in both analyses (Fig. 1 and Online Resource 3), suggesting that $A$. insularis is the non-hexaploid species most closely related to hexaploid oats.

The A-genome diploid species showed the highest overall diversity and formed several well-separated groups. All of the $\mathrm{A}_{\mathrm{s}}$-genome diploid species, with the exception of A. lusitanica, formed a relatively broad cluster (Group 3 ) which could be further divided into two sub-groups in the map-based analysis (Fig. 1): Group 3A contained two $\mathrm{A}_{\mathrm{s}}$-genome species (A. atlantica and A. hirtula), with the rest forming Group 3B. In the de novo analysis (Online Resource 3), the species A. atlantica and A. hirtula were also slightly separated from the remainder of Group 3. Of the remaining A-genome species, the $\mathrm{A}_{1}$-genome species A. longiglumis formed a well-separated group (Group 4), whereas the $\mathrm{A}_{\mathrm{c}}$-genome species $A$. canariensis and the $\mathrm{A}_{\mathrm{d}}$-genome species $A$. damascena formed a single cluster (Group 5), along with two accessions from A. lusitanica.

The AB-genome tetraploids formed two separate clusters (groups 6 and 7) that were distinct from the A-genome groups, but within the same region. Group 6 was comprised 
Fig. 1 Hexaploid-based PCoA analysis depicting the relative distances between hexaploids and other Avena accessions used in this study on the first two axes. Each point represents a sample derived from a single seed from an accession. The number of accessions for each species is shown in Table 1. Some accessions are represented by multiple samples (see Online Resource 1) (colour figure online)

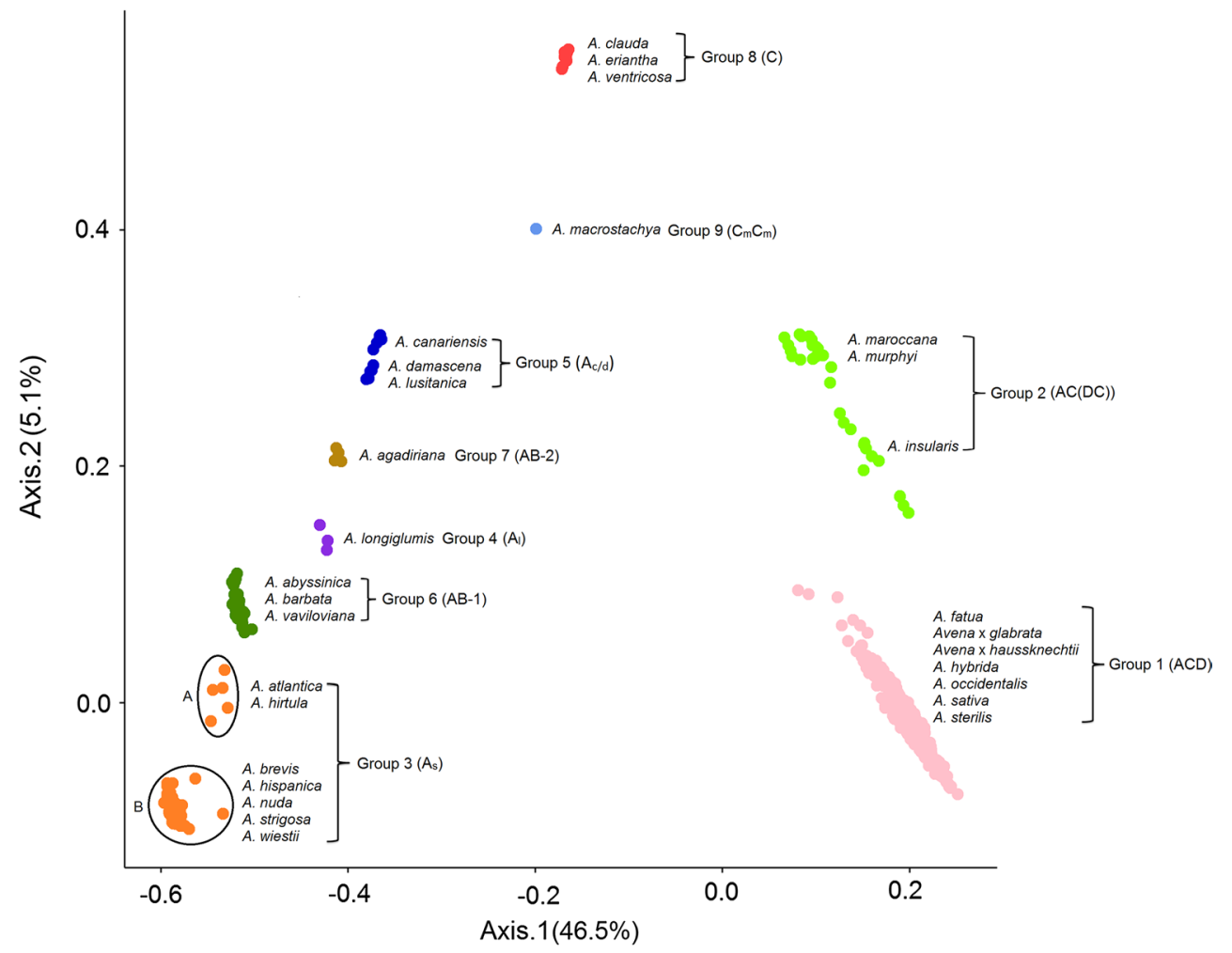

of three species (A. abyssinica, A. barbata, and A. vaviloviana), while Group 7 contained only one species, $A$. agadiriana (Fig. 1). The separation of AB from A genome groups was not as obvious in the de novo analysis, but the two ploidy levels were still separated effectively by the second axis (Online Resource 3).

All three diploid $\mathrm{C}$-genome species fell into a single well-separated cluster (Group 8). This group showed the greatest distance from the $\mathrm{A}_{\mathrm{s}}$ genome (Group 3), based on the first two PCoA axes. The perennial tetraploid species $A$. macrostachya was separated from all other species (Group 9), but showed the closest relationship with the C-genome diploids.

\section{Analysis of in silico polyploids}

Fifty-one possible combinations of diploids with tetraploids were used to create in silico hexaploids, and pair-wise genetic similarities were estimated among all extant and in silico hexaploids. The consistent PCoA patterns shown by multiple species within most ancestral groups provides an effective replication of these results at the ancestralgroup level (Fig. 2). All in silico hexaploids comprised of AC (DC) genome tetraploids plus A-genome diploids clustered closely with the extant hexaploids. The closest in silico matches to the extant hexaploids included the AC (DC) tetraploids plus A. longiglumis, suggesting a possible role of these species in the formation of the hexaploids. The in silico hexaploids comprised of $\mathrm{AB}$ genome tetraploids and $\mathrm{C}$-genome diploids formed a cluster that was more separated from the extant hexaploids. Hierarchical cluster analysis (Online Resource 4) suggested that the extant hexaploids are most closely related to predicted in silico hexaploids comprised of the AC (DC) genome tetraploids and the $\mathrm{A}_{\mathrm{s}}$ or $\mathrm{A}_{1}$ genome diploids.

Thirty-six in silico tetraploids combining all extant A- and C-genome diploids from this study were created to identify putative ancestral diploids involved in the formation of the AC (DC) genome tetraploids. PCoA analysis (Fig. 3) and hierarchical clustering (Online Resource 5) showed that none of the predicted in silico tetraploids clustered together with the extant AC (DC) tetraploids, suggesting that diverged, non-extant forms of the A and/ or $\mathrm{C}$ genomes were involved in the formation of these tetraploids.

\section{Chromosome E-painting by density of shared hexaploid haplotypes}

Unscaled heat maps, based on the sequential map position of each tested locus, show details of shared hexaploid haplotypes within each species and genome group (Online Resource 6, Sheet 1). These heat maps were smoothed and scaled using a sliding $30 \mathrm{cM}$ window (Online Resource 6, Sheet 2). Thresholds were set to allow chromosome E-painting based on relative hexaploid similarity within all ancestral 
Fig. 2 PCoA analysis including all extant hexaploids and theoretical in silico hexaploid species generated by combining tag counts from diploid-plustetraploid combinations. The symbols and colours represent in silico combinations that clustered together, as labelled. The blue "+" symbols represent all extant hexaploid species analysed in this study (colour figure online)

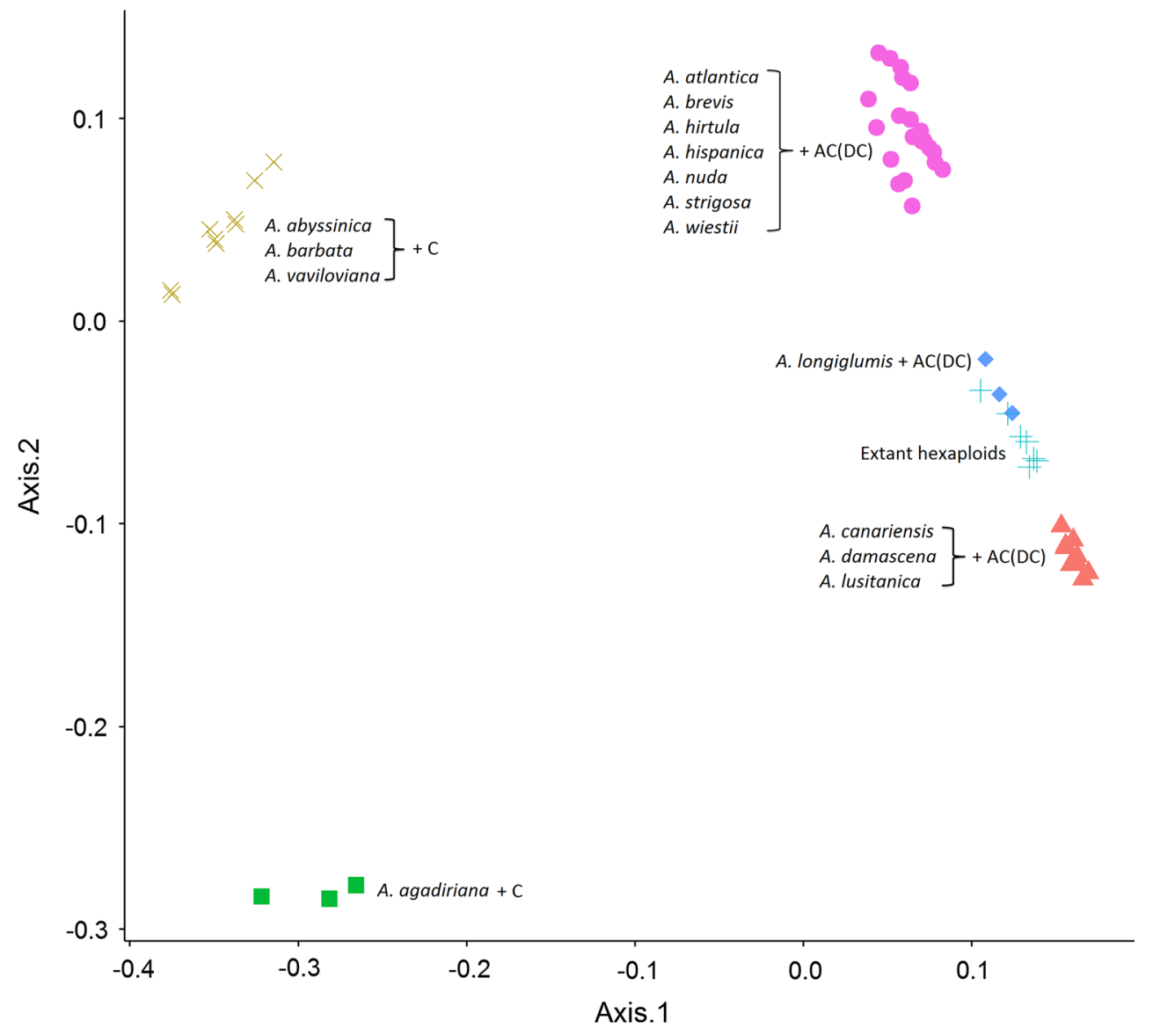

groups (Online Resource 7) and four groups were selected as having the greatest differential resolving power among hexaploid chromosome representations (Fig. 4). We emphasize here that the thresholds are set independently for each ring in the above figures, since the overall similarity of some groups is much higher than others (Table 1) and the intention here is to observe patterns of relative similarity within each group.

In Fig. 4 and Table 2, we have interpreted the painted chromosome representations by ordering them as follows: chromosomes with Mrg identifiers 5, 12, 20, 23, 24, 33, and part of 18 showed the strongest relative matches in the $\mathrm{A}_{\mathrm{s}}-$ genome diploid species. These same chromosomes showed weak relative matches in the $\mathrm{C}$-genome species and also in the AC (DC) group. Table 2 illustrates that this group of chromosomes is consistent with those that have been confirmed by monosomic assignment as being A-genome chromosomes. Chromosomes with Mrg numbers 3, 9, 11, $13,15,17$, and parts of $18,19,6,8,21$, and 28 showed the weakest matches to $\mathrm{A}_{\mathrm{s}}$-genome diploids, and the strongest matches to $\mathrm{C}$-genome diploids and the $\mathrm{AC}$ (DC) group. Table 2 illustrates that this group of chromosomes is consistent with those that have been confirmed by monosomic assignment as being C-genome chromosomes. Chromosomes with Mrg numbers 1, 2, and 4, as well as parts of
$19,6,8,21$, and 28 showed relatively weak similarities to $\mathrm{A}$ and $\mathrm{C}$ genome diploids, but strong similarities to the $\mathrm{AC}$ (DC) tetraploids. Table 2 illustrates that this group of chromosomes is consistent with those that have been confirmed by monosomic assignment as being D-genome chromosomes. In Online Resource 7, it can be seen that all other A-genome groups, as well as the AB genome groups, follow a similar pattern to that of $\mathrm{A}_{\mathrm{s}}$.

\section{Chromosome E-painting by haplotype diversity}

The highest diversity of hexaploid haplotypes was observed in the AC (DC) group, with an average of 1.24 haplotypes for those loci where any hexaploid haplotype was present (Table 1). This was much higher than the diversity seen in any other group, with the exception of the ACD genome group in which the haplotypes were originally discovered. Of the AC (DC)-genome tetraploids, the species $A$. insularis shared more hexaploid alleles (1.14 alleles per locus present) than either A. maroccana (1.06) or A. murphyi (1.00) (Table 1). These data were also interpreted using a circle diagram, with independent thresholds for each ancestral group to show relative patterns across chromosomes (Online Resource 8). Although not as striking as 
Fig. 3 PCoA analysis including all extant AC (DC) tetraploids and theoretical in silico tetraploid species generated by combining tag counts from A-genome diploid plus C-genome diploid combinations. The symbols and colours represent in silico combinations that clustered together, as labelled. The green triangles represent all extant AC (DC) tetraploid species analysed in this study (colour figure online)

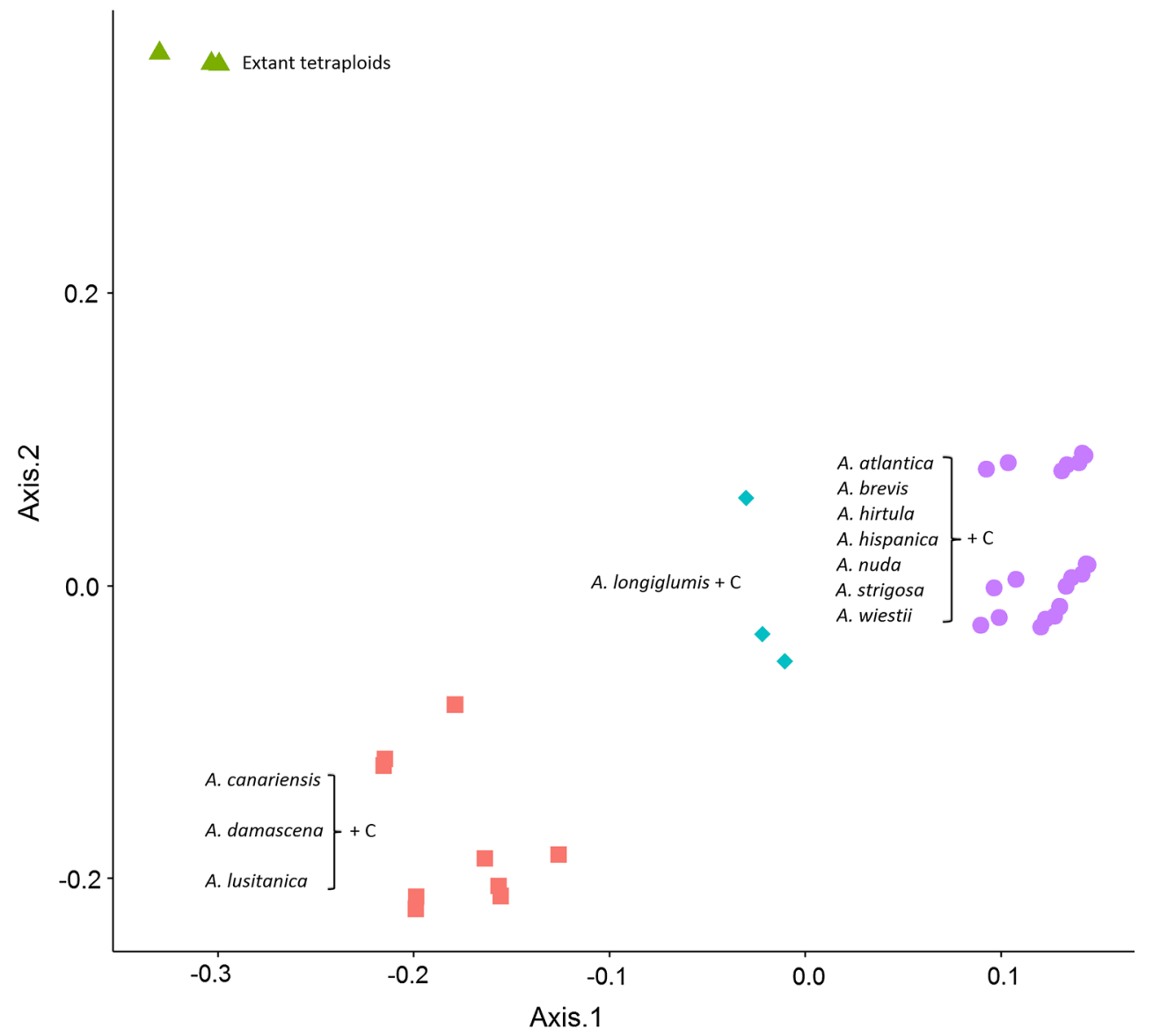

the patterns seen for locus presence (Fig. 4), the diversity of hexaploid haplotypes followed a similar pattern that matched the genome assignments made earlier.

\section{Discussion}

This work represents one of the largest and most comprehensive genomic studies of the genus Avena to date, and has generated the following novel results, which are discussed below: (1) support for the definitive classification of DC-genome tetraploids, (2) a map-level identification of the ancestral origin of 21 hexaploid chromosomes, (3) confirmation that no extant A-genome diploids should be considered as D-genome diploids, and (4) evidence for the continued classification of a diverged $\mathrm{B}$ genome in $\mathrm{AB}$ tetraploids. We begin with a discussion to support the chosen methods of data analysis.

\section{Justification and comparison of two methods of GBS analysis}

Although molecular markers have the capacity to estimate both within- and among-species diversity, with genome coverage that is superior to single-gene methods, it is recognized that GBS and other marker technologies present limitations and potential sources of bias relative to alignment-based phylogeny analyses (DaCosta and Sorenson 2016; Qi et al. 2015). In particular, the use of GBS in the absence of a reference genome may confound intra- vs. inter-locus polymorphism, and the filtering of polymorphisms based on allele or heterozygote frequency could also introduce bias. Since we are not attempting to estimate magnitudes of divergence, we have accepted a pragmatic tolerance of these factors. Furthermore, the methods that we have selected are deliberately focused on distinguishing among species that contain a series of homeologous genomes, such that variation among paralogous loci must be incorporated. We also attempted to address potential sources of bias using two different methods of GBS analysis; thus, providing two independent assessments of marker-based diversity. In one analysis, we calculated genetic similarity based on the presence of tag-level haplotypes that were mapped in the hexaploid genome. In the second analysis, we attempted to avoid potential ascertainment bias by estimating similarities based on the sharing of key GBS tag-level haplotypes. In this analysis, we mitigated the problem of allele dropouts by combining and 


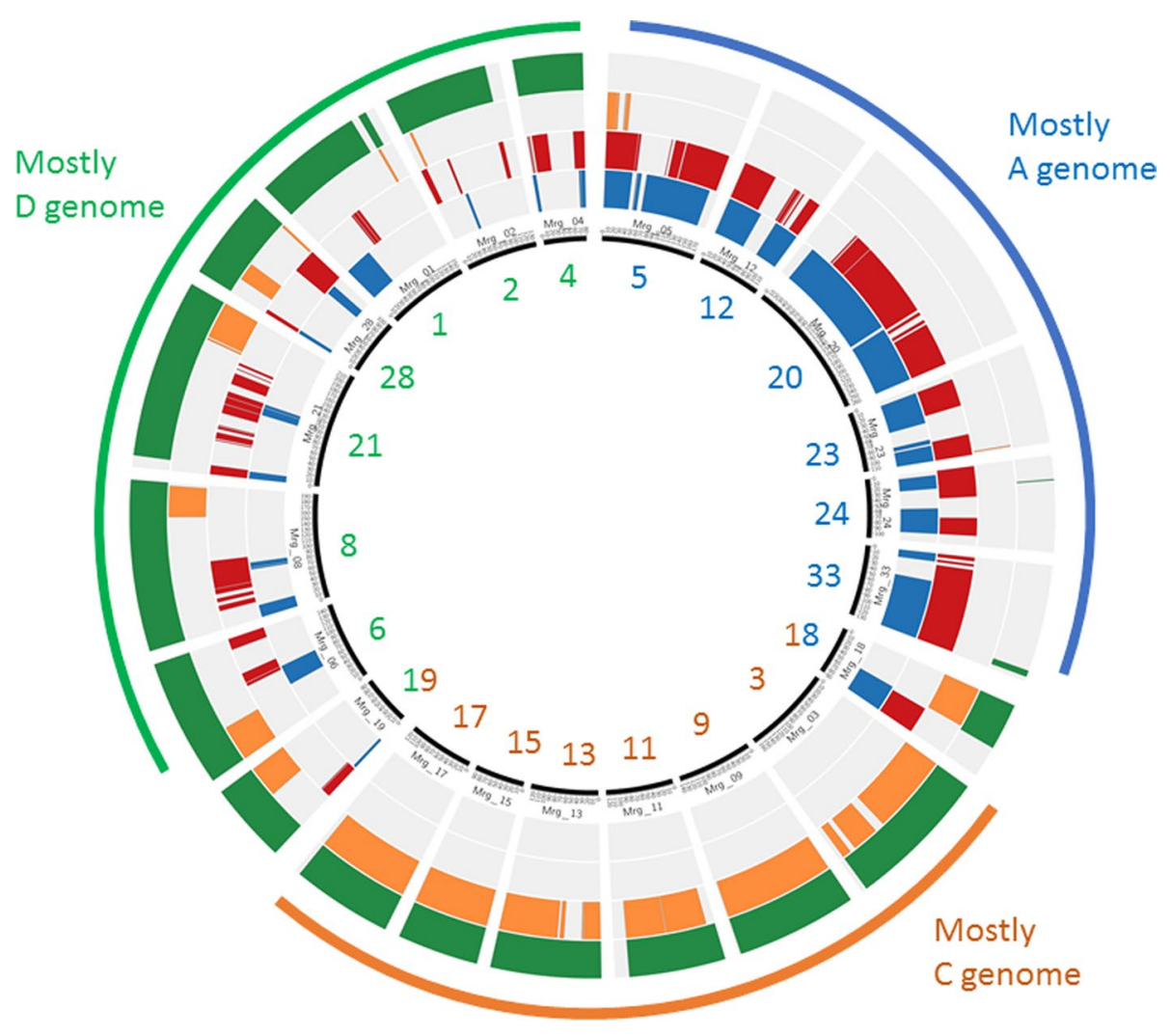

Fig. 4 E-painting of chromosome representations in the hexaploid oat consensus map. The 21 chromosome representations with Mrg identification numbers described by Chaffin et al. (2016) are shown as elements of the circle, scaled by $\mathrm{cM}$ distance, and ordered by inferred genome origin. Four concentric rings of different colours indicate which chromosomes within the map contain a relatively high frequency of matches to the ancestral group represented by the respective ring: blue (innermost ring) represents A-genome diploids; red (second ring) represents the $\mathrm{A}_{\mathrm{c} / \mathrm{d}}$-variant genome of the group containing A. canariensis, A. damascena and A. lusitanica; orange (third ring) represents $\mathrm{C}$-genome diploids; and green (outermost ring) represents AC (DC)-genome tetraploids. The three outermost arcs are an interpretation of the genome contributions to the majority of chromosomes within each arc, based on hybridization pattern to the ancestral genomes (colour figure online) normalizing tag counts by species, to achieve a high effective sampling depth. The fact that both methods produced clear and qualitatively consistent separation among species should lend credibility to those parts of our discussion that relate to phylogeny.

It is interesting that the map-based hexaploid analysis produced greater separation among many species than did the de novo discovery method. We had expected to find better separation using the latter method, which should be more inclined to identify differences based on unique haplotypes found only in one species. One potential explanation for our result is that some A. sativa oat varieties contain introgressions from other species (e.g., Aung et al. 2010; Hoppe and Kummer 1991; Rines et al. 2007); thus, it is likely that some mapped hexaploid haplotypes have recent origins in other species. However, this would probably account for only a small number of introgressions from A. sterilis, A. strigosa, and A. maroccana, and could not account (for example) for the improved separation seen between A- vs. AB-genome species in the hexaploid GBS model. A more likely explanation is that the strong separation among species in the hexaploid analysis is caused primarily by differing degrees of overall similarity to the hexaploid genome. This would also explain why the clusters in the hexaploid model (Fig. 1) are positioned along a continuous arc, reflecting a gradient of similarity on two axes, whereas the clusters in the de novo model (Online Resource 3) follow a less linear pattern. It is likely that the two axes in Fig. 1 are correlated with markers from the A or $\mathrm{D}$ genomes (axis 1) and the $\mathrm{C}$ genome (axis 2).

\section{The ancestral $D$ genome of hexaploid oat can be found in three tetraploid species}

We consider the most important result from this work to be three strong lines of evidence that the $\mathrm{C}$ and $\mathrm{D}$ genome of hexaploid oat originated from an ancestor that is similar to the three extant DC genome (formerly AC) tetraploid species. 
Table 2 Previous and current physical assignment of 21 chromosome representations from the hexaploid oat consensus map

\begin{tabular}{|c|c|c|c|c|}
\hline Consensus chromosome ${ }^{\mathrm{a}}$ & Confirmed assignment ${ }^{\mathrm{a}}$ & Previous assignment ${ }^{b}$ & Diploid assignment $^{\mathrm{c}}$ & New genome assignment ${ }^{\mathrm{d}}$ \\
\hline $\operatorname{Mrg} 05$ & $16 \mathrm{~A}$ & $\mathbf{1 6 A}, 1 \mathrm{C}$ & $\mathrm{AD}$ & A \\
\hline $\operatorname{Mrg} 12$ & & $13 \mathrm{~A}$ & $\mathrm{AD}$ & A \\
\hline $\operatorname{Mrg} 20$ & & $19 \mathrm{~A}$ & $\mathrm{AD}$ & A \\
\hline $\operatorname{Mrg} 23$ & $11 \mathrm{~A}$ & $11 \mathrm{~A}$ & $\mathrm{AD}$ & A \\
\hline $\operatorname{Mrg} 24$ & & 8A (14D) & $\mathrm{AD}$ & A \\
\hline $\operatorname{Mrg} 33$ & & $15 \mathrm{~A}$ & $\mathrm{AD}$ & A \\
\hline $\operatorname{Mrg} 18$ & & $7 \mathrm{C}-17 \mathrm{~A}$ & $\mathrm{C}$ & C/A \\
\hline $\operatorname{Mrg} 03$ & $4 \mathrm{C}$ & $4 C(10 D)$ & $\mathrm{C}$ & $\mathrm{C}$ \\
\hline Mrg09 & $6 \mathrm{C}$ & 6C & $\mathrm{C}$ & $\mathrm{C}$ \\
\hline $\operatorname{Mrg} 11$ & & $1 \mathrm{C}$ & $\mathrm{C} / \mathrm{A}$ & $\mathrm{C}$ \\
\hline $\operatorname{Mrg} 13$ & & (20D) & $\mathrm{C}$ & $\mathrm{C}$ \\
\hline $\operatorname{Mrg} 15$ & $2 \mathrm{C}$ & $\mathbf{2 C}(10 \mathrm{D})$ & $\mathrm{C}$ & $\mathrm{C}$ \\
\hline $\operatorname{Mrg} 17$ & & $3 \mathrm{C}$ & $\mathrm{C}$ & $\mathrm{C}$ \\
\hline $\operatorname{Mrg} 19$ & $21 \mathrm{D}$ & 21D & C/AD & $\mathrm{C} / \mathrm{D}$ \\
\hline Mrg06 & & $14 \mathrm{D}$ & $\mathrm{AD} / \mathrm{C}$ & $\mathrm{D} / \mathrm{C}$ \\
\hline $\operatorname{Mrg} 08$ & $12 \mathrm{D}$ & 12D & $\mathrm{AD} / \mathrm{C}$ & $\mathrm{D} / \mathrm{C}$ \\
\hline $\operatorname{Mrg} 21$ & & $(16 \mathrm{~A})$ & $\mathrm{AD} / \mathrm{C}$ & $\mathrm{D} / \mathrm{C}$ \\
\hline $\operatorname{Mrg} 28$ & & $7 \mathrm{C}-(17 \mathrm{~A})$ & $\mathrm{C}$ & $\mathrm{D} / \mathrm{C}$ \\
\hline $\operatorname{Mrg01}$ & & $(5 \mathrm{C})$ & $\mathrm{AD}$ & $\mathrm{D}$ \\
\hline $\mathrm{Mrg02}$ & 9D & 9D & $\mathrm{AD}$ & $\mathrm{D}$ \\
\hline $\operatorname{Mrg} 04$ & $18 \mathrm{D}$ & $18 \mathrm{D}$ & $\mathrm{AD}$ & $\mathrm{D}$ \\
\hline
\end{tabular}

a Consensus chromosome representation and confirmed assignments are based on Chaffin et al. (2016)

b Chromosome assignments by Oliver et al. (2013) were based on an earlier consensus map with fewer markers. Bold font assignments are now confirmed, those in parentheses are probably incorrect, and those remaining were not confirmed but are not disputed

${ }^{c}$ Diploid genome assignment was performed by Chaffin et al. (2016) based on sequence matches to draft shotgun genome sequences of A- and $\mathrm{C}$-genome diploid oats. This method was unable to resolve A and D genome chromosomes. Where assignments are split by a forward slash, the assignment given to the longest part of the chromosome is shown first

${ }^{\mathrm{d}}$ Inferred genome assignment based on the current study. Where assignments are split, suggesting a large inter-genome translocation, the assignment given to the longest part of the chromosome is shown first. Possible inter-genome translocations smaller than $15 \%$ of a chromosome are not shown in this table but can be inferred based on Fig. 1

First, our results from the analysis of in silico polyploids showed that complementation of the AC (DC) genomes with any A-genome diploid resulted in close theoretical matches to the extant ACD hexaploids. Moreover, these matches were much better than any match involving two A genomes plus a $\mathrm{C}$ genome. If the tetraploids contained an $\mathrm{AC}$ genome, the match of hexaploids to $\mathrm{AC}+\mathrm{A}$ would not have been substantially better than the matches to $\mathrm{A}+\mathrm{A}+\mathrm{C}$.

The second line of evidence is provided by map-based chromosome E-painting. This approach was effective likely because most GBS tags originate in non-coding DNA (Huang et al. 2014) that is subject to rapid divergence among genomes that have become separated by reproductive barriers. Thus, the A and D genomes could be distinguished by a composite analysis of GBS tags more effectively than by single conserved gene sequences. In the analysis depicted by Fig. 4, approximately $1 / 3$ of the hexaploid chromosomes matched relatively poorly to A- and
C-genome diploids, but showed strong matches to the three AC (DC) tetraploid species. These were designated as D-genome chromosomes. Conversely, the 1/3 of chromosomes not matched by the AC (DC) tetraploids showed strong matches to the A-genome diploids, and were designated as A-genome chromosomes. These assignments were consistent with all previously confirmed physical assignments made by Chaffin et al. (2016) confirming that the AC (DC) tetraploids contain a $\mathrm{D}$, and not an $\mathrm{A}$, genome.

A third line of evidence is that the diversity of hexaploid alleles in AC (DC) tetraploids was higher than for any other species, especially for the chromosomes determined to be of $\mathrm{C}$ - and $\mathrm{D}$-genome origin in the above analysis (Online Resource 8). This also suggests that the ACD and DC species share a recent common DC ancestor, and possibly, that multiple polyploidization events occurred to maintain the multiple shared haplotypes that were observed at many loci. 
All of the above results support a final re-designation of A. insularis, A. maroccana, and A. murphyi as DC genomes. This would reflect a true A vs. D differentiation in hexaploid genomes, as well as a recognition of the probable common lineage of DC and ACD species to an ancestral D genome that is substantially diverged from extant A genomes. It is recognized (and discussed later) that the D genome is related to the A genome, and that it may have diverged from A-genome ancestors within the polyploid species. However, this proposition, together with our results showing a clear similarity of D-genome chromosomes between the DC and ACD genome species, provides evidence for a sequence of polyploidization events where the A genome is the most recent hexaploid addition to a common DC-genome ancestor.

This is not the first suggested use of the DC genome designation, nor the first evidence that has been presented for this nomenclature (Yan et al. 2014). However, most previous reports, including the recent report by Chew et al. (2016), have continued to identify the tetraploids as AC genomes. This classification fails to identify the closest ancestry of the D genome chromosomes that are recognized in hexaploid oat. Our new evidence and call for the DC genome designation is relevant and important for ongoing germplasm conservation efforts, as well as for future efforts in pre-breeding and cultivar development. It means that the D genome is available in extant non-hexaploid wild relatives for comparative genome analysis and allele mining. Many QTL in cultivated oat have been reported on chromosomes that correspond with those from the D genome, including the N1 locus responsible for primary variation in hull retention (De Koeyer et al. 2004), now known to be on $\mathrm{Mrg} 21$, and a major flowering time QTL on Mrg02 (Klos et al. 2016). Preservation, expansion, and utilization of DC genome tetraploids, especially A. insularis, which showed the highest number and diversity of hexaploid haplotypes, should be considered a high priority.

\section{Physical chromosome assignment in the hexaploid consensus map is confirmed and improved}

Our chromosome E-painting results have also contributed to a substantial improvement in physical chromosome assignment in the hexaploid linkage map (Table 2). Here, we use the most recent nomenclature for physical chromosomes (Sanz et al. 2010), which merges two previous physical nomenclatures, compared to the consensus map nomenclature of Chaffin et al. (2016). We have shown that all of the nine validated chromosome assignments are consistent with the current analysis; thus, we have used the current analysis to assign genome designations to all of the remaining chromosomes. This includes the designation of two important homeologous chromosomes that were previously ambiguous: $\mathrm{Mrg} 20$ is from the A genome and Mrg21 is mostly from the D genome.

Because these assignments were made within chromosomes on a map-based level (Fig. 4), we can also evaluate some of the genome restructuring that has taken place within the hexaploid genome. The majority of translocations appear to involve the $\mathrm{D}$ and $\mathrm{C}$ genomes, possibly as non-reciprocal translocations, since there appear to be six intact $\mathrm{C}$ chromosomes vs. three $\mathrm{D}$ chromosomes that do not contain significant translocations from the $\mathrm{C}$ genome. This observation is consistent with FISH results reported by Irigoyen et al. (2002) and Sanz et al. (2010), who both observed a majority of C- to D-genome translocations. The chromosome representations Mrg18 and Mrg28 were previously assigned as the reciprocal translocations 7C-17A/17A-7C (Oliver et al. 2013), although this designation was not confirmed by Chaffin et al. (2016). Of these, only Mrg 18 contains a clear A/C translocation, while Mrg28 appears to contain a C/D translocation. While it is possible that non-balanced $\mathrm{A} / \mathrm{C} / \mathrm{D}$ translocations might have occurred, this substantial change in interpretation will require further evaluation. The preponderance of $\mathrm{C} / \mathrm{D}$ vs. $\mathrm{C} / \mathrm{A}$ or $\mathrm{A} / \mathrm{D}$ translocations may provide further evidence that the $\mathrm{C}$ and $\mathrm{D}$ genomes joined first to form a DC tetraploid, and that the A genome of the hexaploid was introduced much later, possibly after the $\mathrm{C}$ and $\mathrm{D}$ genomes in the tetraploid had co-evolved, and after the A and D genomes had diverged substantially from a common ancestor. However, additional factors may also have affected the differential fragmentation of the three hexaploid genomes. One such factor may be the tolerance of each genome to fragmentation, which may relate to systematic changes in expression and suppression that took place following polyploidization events. Such factors require further investigation.

While this map-level analysis of translocations may provide valuable information for future genome analysis and sequence assembly, it should also be emphasized that: (1) this consensus map does not (by definition) represent the genome configuration of all oat varieties (Chaffin et al. 2016), (2) errors and uncertainties are present in all linkage maps, and (3) the presence of translocations is known to be heterogeneous among oat varieties (Irigoyen et al. 2002; Jellen and Beard 2000; Sanz et al. 2010).

\section{Diploid species containing the A genome are diverse, but none contain the hexaploid $D$ genome}

At least twelve diploid species containing the A genome have been proposed to be part of the genus Avena, of which we have studied eleven. Our results showed this to be the most diverse set of species within a single genome designation (Fig. 1 and Online Resource 3), which agrees with 
the broad geographic distribution of the A-genome diploids (Baum 1977). Of interest is the result from in silico polyploid analysis suggesting that $A$. longiglumis could be the A genome donor, combined with a $\mathrm{CD}$ genome from one of the tetraploids (Fig. 2). Separate groups within the A-genome designation also matched well with the presence of previously reported structural differences among these genome types. The species in Group 5, including $A$. canariensis as well as some A. damascena and A. lusitanica accessions, showed the greatest divergence from other groups (Fig. 1 and Online Resource 3). The two species $A$. canariensis and $A$. damascena form hybrids with regular bivalent pairing in most pollen mother cells (Leggett 1984) and, although A. lusitanica is often classified as having an $\mathrm{A}_{\mathrm{s}}$ genome, this was questioned by Thomas (1992) and its clustering here, rather than with other $\mathrm{A}_{\mathrm{s}}$ species, may support a future reclassification. Both A. canariensis and $A$. damascena have been proposed to be the $\mathrm{D}$-genome donors of the hexaploid species, not only because of the absence of an A-genome specific DNA repeat (Linares et al. 1998), but also because of considerable divergence from other A-genome diploid species (Baum et al. 1973; Rajhathy and Baum 1972). While this divergence is supported strongly by our results, our map-based analysis of hexaploid haplotype identity suggests that the $A_{c}$ and $A_{d}$ genomes are much closer to the hexaploid A genomes than they are to the hexaploid D genomes (Fig. 4). Our in silico tetraploid analysis (Fig. 3) supports this conclusion.

\section{Diploid species containing the $\mathrm{C}$ genome are genetically homogeneous}

There are three diploid species considered to carry the C genome. All three of these were included in the Section Ventricosa (Baum 1977). On the basis of karyotype, they were further divided into two genome types: $\mathrm{C}_{\mathrm{p}}($ A clauda and $A$. eriantha) and $\mathrm{C}_{\mathrm{v}}(A$. ventricosa) (Leggett and Thomas 1995; Rajhathy and Thomas 1967). In this study, all three $\mathrm{C}$-genome diploid species were tightly clustered based on two PCoA axes (Fig. 1 and Online Resource 3), suggesting a high degree of genetic homogeneity. This was consistent with other molecular studies (Drossou et al. 2004; Yan et al. 2014), with the fertility of interspecific hybrids (Rajhathy and Thomas 1967), and with additional karyotype studies (Fominaya et al. 1988; Rajhathy and Thomas 1967). The geographical distribution of this group may also explain the high degree of genetic homogeneity among these species. All species within this group are restricted to the Mediterranean shoreline (Baum 1977). Both $\mathrm{C}_{\mathrm{p}}$-genome species are distributed in nearly identical regions around the Mediterranean, while the range of the $\mathrm{C}_{\mathrm{v}}$-genome diploid A. ventricosa is within that of the
$\mathrm{C}_{\mathrm{p}}$-genome species (Rajhathy and Thomas 1967). Other than through morphological differences (Baum 1977), it would be difficult to support the current classification of three $\mathrm{C}$-genome species.

\section{The B genome has diverged from the A genome and there are two distinct $\mathrm{AB}$-genome groups}

Our results are in agreement with most other literature in suggesting that the $\mathrm{B}$ genome within the $\mathrm{AB}$-genome tetraploids is sufficiently different from the A genome to cause a distinct clustering of AB-genome species. However, Chew et al. (2016) observed that each of two different ABgenome groups coincided with an A-genome group. We have since determined that the majority of the A-genome members of their Group 4A (including CN 25414, PI 657427, PI 657472) are probably misclassified tetraploids (see Online Resource 1); thus, it is possible that this group is exclusively a tetraploid group. Such misclassifications are usually initiated by the people who originally submitted the accessions to a genebank, and are not the fault of the genebank curator or the users through whom these errors propagate. Nevertheless, while we consider that an A vs. $\mathrm{B}$ distinction may still be warranted, we agree that the $\mathrm{B}$ genome is likely to be a diverged form of the A genome, and that close relatives of both the A and B genomes may be found within the A genome group. Such work may require further genotyping, since our efforts to identify components of the $\mathrm{AB}$ genome through in silico methods were inconclusive (data not shown).

In this study, A. agadiriana was separated from the other $\mathrm{AB}$ genome tetraploids (Fig. 1), which confirms previously reported structural differences. However, A. agadiriana was substantially more similar to the $\mathrm{AB}$ group than it was to the AC (DC) group. Furthermore, based on E-painting (Online Resource 7), A. agadiriana appears similar only to the A genome of hexaploid oat and not to the D genome, in the same manner as the other $\mathrm{AB}$ genome tetraploids. Because of the proximity of A. agadiriana to the group containing A. canariensis, we tentatively propose that it may share one of its two genomes with this group. The existence of two distinct AB-genome groups, and their similarity to A-genome diploid groups, suggests that further detailed work should be performed to gain insight into the formation of these tetraploids.

\section{Further research possibilities}

Through this work, we have demonstrated that GBS analysis is effective in the qualitative delineation of genome similarities at multiple levels, including ancestral groups, species, and accessions within species. Although this work 
has focussed primarily on distinctions at the species level, we noted in the development of these methods that these data could be reanalysed to focus on greater resolution within each species, and we intend to pursue this in forthcoming work. In addition, we have demonstrated a method by which genome origins can be effectively delineated at a map-based level in hexaploid oat. This method may be useful in other complex polyploids for which genome sequences are not yet available. We found that GBS analysis was robust, with two different approaches giving similar results at this level of exploration. However, we caution users regarding potential pitfalls in GBS analysis, including ascertainment bias caused by filtering and estimation bias in GBS allele frequencies for phylogenetic analysis. While GBS was effective for the purposes of this work, additional research is required to calibrate estimates of similarity based on GBS with evolutionary distance. Such work will probably require the assignment of GBS tags to draft genome sequences, permitting the alignment and analysis of long and accurate GBS haplotypes. We also suggest that GBS analysis may contribute sequence data that could be used to design new genome- or chromosome-specific probes, allowing for further refinement of physical maps. Finally, this work underscores the need for high-quality internationally accessible germplasm collections. Our work benefited from excellent collections of Avena species housed in Canadian and American germplasm banks. Despite this, we have identified one species of paramount interest, $A$. insularis, which today is represented by only three known accessions.

Author contribution statement HY: conducted experiments, analysed the data and co-wrote the manuscript; WAB: contributed to data analysis and interpretation of results; CPW: contributed to design and execution of experiments and interpretation of results; YP, TL RGL, $\mathrm{Y}-\mathrm{BF}, \mathrm{AD}, \mathrm{CJH}, \mathrm{ENJ}$ : contributed to experimental design, interpretation of results, and revisions of manuscript; BB: constructed GBS libraries and provided key advice on GBS analysis; YW, YP, and NAT: supervised HY; NAT conceived the study, analysed the data, and co-wrote the manuscript. All authors read and approved the final manuscript.

\footnotetext{
Acknowledgments We gratefully acknowledge the professional and technical assistance provided by Abakar Kaka, Dallas Kessler, Travis Sander, Gregory Peterson, Carolee Horbach, Kathy Upton, Biniam Hizbai, and Pingping Zhou.
}

\section{Compliance with ethical standards}

The authors declare no breaches of ethical standards.
Funding This work was funded by the Canadian Crop Genomics Initiative of the Government of Canada as part of core Agriculture and Agri-Food Canada funding.

Conflict of interest The authors declare that they have no conflict of interest.

Open Access This article is distributed under the terms of the Creative Commons Attribution 4.0 International License (http://creativecommons.org/licenses/by/4.0/), which permits unrestricted use, distribution, and reproduction in any medium, provided you give appropriate credit to the original author(s) and the source, provide a link to the Creative Commons license, and indicate if changes were made.

\section{References}

Alicchio R, Aranci L, Conte L (1995) Restriction fragment length polymorphism based phylogenetic analysis of Avena L. Genome 38:1279-1284

Aung T, Zwer P, Park R, Davies P, Sidhu P, Dundas I (2010) Hybrids of Avena sativa with two diploid wild oats (CIav6956) and (Clav7233) resistant to crown rust. Euphytica 174:189-198

Badaeva ED, Shelukhina OY, Goryunova SV, Loskutov IG, Pukhalskiy VA (2010) Phylogenetic relationships of tetraploid ABgenome Avena species evaluated by means of cytogenetic (C-banding and FISH) and RAPD analyses. J Bot 2010:1-13

Baum BR (1977) Oats: wild and cultivated. A monograph of the genus Avena L. (Poaceae). Minister of Supply and Services, Ottawa

Baum BR, Fedak G (1985a) Avena atlantica, a new diploid species of the oat genus from Morocco. Can J Bot 63:1057-1060

Baum BR, Fedak G (1985b) A new tetraploid species of Avena discovered in Morocco. Can J Bot 63:1379-1385

Baum BR, Rajhathy T, Sampson DR (1973) An important new diploid Avena species discovered on the Canary Islands. Can J Bot 51:759-762

Chaffin AS, Huang Y-F, Smith S, Bekele WA, Babiker E, Gnanesh BN, Foresman BJ, Blanchard SG, Jay JJ, Reid RW, Wight CP, Chao S, Oliver R, Islamovic E, Kolb FL, McCartney C, Mitchell Fetch JW, Beattie AD, Bjørnstad Å, Bonman JM, Langdon T, Howarth CJ, Brouwer CR, Jellen EN, Klos KE, Poland JA, Hseih T-F, Brown R, Jackson E, Schlueter JA, Tinker NA (2016) A consensus map in cultivated hexaploid oat reveals conserved grass synteny with substantial subgenome rearrangement. Plant Genome. doi:10.3835/plantgenome2015.10.0102

Chen Q, Armstrong K (1994) Genomic in situ hybridization in Avena sativa. Genome 37:607-612

Chew P, Meade K, Hayes A, Harjes C, Bao Y, Beattie AD, Puddephat I, Gusmini G, Tanksley SD (2016) A study on the genetic relationships of Avena taxa and the origins of hexaploid oat. Theor Appl Genet 129:1405-1415

Craig IL, Murray BE, Rajhathy T (1974) Avena canariensis: morphological and electrophoretic polymorphism and relationship to the A. magna-A. murphyi complex and A. sterilis. Can J Bot 16:677-689

DaCosta JM, Sorenson MD (2016) ddRAD-seq phylogenetics based on nucleotide, indel, and presence-absence polymorphisms: analyses of two avian genera with contrasting histories. Mol Phylogenet Evol 94:122-135 
De Koeyer DL, Tinker NA, Wight CP, Deyl J, Burrows VD, O'Donoughue LS, Lybaert A, Molnar SJ, Armstrong KC, Fedak G, Wesenberg DM, Rossnagel BG, McElroy AR (2004) A molecular linkage map with associated QTLs from a hulless $\mathrm{x}$ covered spring oat population. Theor Appl Genet 108:1285-1298

Drossou A, Katsiotis A, Leggett JM, Loukas M, Tsakas S (2004) Genome and species relationships in genus Avena based on RAPD and AFLP molecular markers. Theor Appl Genet 109:48-54

Fominaya A, Vega C, Ferrer E (1988) Giemsa C-banded karyotypes of Avena species. Genome 30:627-632

Fu Y-B, Williams DJ (2008) AFLP variation in 25 Avena species. Theor Appl Genet 117:333-342

Hayasaki M, Morikawa T, Tarumoto I (2000) Intergenomic translocations of polyploid oats (genus Avena) revealed by genomic in situ hybridization. Genes Genet Syst 75:167-171

Hoppe H, Kummer M (1991) New productive hexaploid derivatives after introgression of Avena pilosa features. Vortraege fuer Pflanzenzuechtung 20:56-61 (Germany)

Huang Y-F, Poland JA, Wight CP, Jackson EW, Tinker NA (2014) Using genotyping-by-sequencing (GBS) for genomic discovery in cultivated oat. PLoS One 9:e102448

Irigoyen M, Loarce Y, Linares C, Ferrer E, Leggett M, Fominaya A (2001) Discrimination of the closely related A and B genomes in AABB tetraploid species of Avena. Theor Appl Genet 103:1160-1166

Irigoyen M, Linares C, Ferrer E, Fominaya A (2002) Fluorescence in situ hybridization mapping of Avena sativa L. cv. Sun II and its monosomic lines using cloned repetitive DNA sequences. Genome 45:1230-1237

Jellen EN, Beard J (2000) Geographical distribution of a chromosome $7 \mathrm{C}$ and 17 intergenomic translocation in cultivated oat. Crop Sci 400:256-263

Jellen EN, Gill BS (1996) C-banding variation in the Moroccan oat species Avena agadiriana $(2 n=4 \times=28)$. Theor Appl Genet 92:726-732

Jellen EN, Phillips RL, Rines HW (1993) C-banded karyotypes and polymorphisms in hexaploid oat accessions (Avena spp.) using Wright's stain. Genome 36:1129-1137

Jellen EN, Gill BS, Cox TS (1994) Genomic in situ hybridization differentiates between $\mathrm{A} / \mathrm{D}$-and $\mathrm{C}$-genome chromatin and detects intergenomic translocations in polyploid oat species (genus Avena). Genome 37:613-618

Kihara H, Nishiyama I (1932) Different compatibility in reciprocal crosses of Avena, with special reference to tetraploid hybrids between hexaploid and diploid species. Jpn J Bot 6:245-305

Klos KE, Huang Y-F, Bekele WA, Obert DE, Babiker E, Beattie AD, Bjørnstad A, Bonman JM, Carson ML, Chao S, Gnanesh BN, Grifiths I, Harrison SA, Howarth CJ, Hu G, Ibrahim A, Islamovic E, Jackson E, Jannink JL, Kolb FL, McMullen MS, Mitchell Fetch JW, Murphy JP, Ohm HW, Rines HW, Rossnagel BG, Schlueter JA, Sorrells ME, Wight CP, Yan W, Tinker NA (2016) Population genomics related to adaptation in elite oat germplasm. The Plant Genome First Look (https://dl.sciencesocieties. org/publications/tpg/first-look)

Krzywinski M, Schein J, Birol I, Connors J, Gascoyne R, Horsman D, Jones SJ, Marra MA (2009) Circos: an information aesthetic for comparative genomics. Genome Res 19:1639-1645

Ladizinsky G (1969) New evidence on the origin of the hexaploid oats. Evolution 23:676-684

Ladizinsky G (1971) Chromosome relationships between tetraploid $(2 n=28)$ Avena murphyi and some diploid, tetraploid and hexaploid species of oats. Can J Bot 13:203-209

Ladizinsky G (1994) The taxonomic status of Avena magna-reappraisal. Lagascalia 17:325-328
Ladizinsky G (1998) A new species of Avena from Sicily, possibly the tetraploid progenitor of hexaploid oats. Genet Resour Crop Evol 45:263-269

Ladizinsky G (2012) Studies in oat evolution: a man's life with Avena. Springer, Heidelberg

Leggett JM (1984) Morphology and metaphase chromosome pairing in three Avena hybrids. Can J Genet Cytol 26:641-645

Leggett JM (1989) Interspecific diploid hybrids in Avena. Genome 32:346-348

Leggett JM, Thomas H (1995) Oat evolution and cytogenetics. In: Welch RW (ed) The Oat Crop. Springer, Heidelberg, pp 120-149

Leggett JM, Thomas HM, Meredith MR, Humphreys MW, Morgan WG, King IP (1994) Intergenomic translocations and the genomic composition of Avena maroccana Gdgr. revealed by FISH. Chromosome Res 2:163-164

Li C-D, Rossnagel BG, Scoles GJ (2000) Tracing the phylogeny of the hexaploid oat with satellite DNAs. Crop Sci 40:1755-1763

Linares C, González J, Ferrer E, Fominaya A (1996) The use of double fluorescence in situ hybridization to physically map the positions of 5S rDNA genes in relation to the chromosomal location of 18S-5.8S-26S rDNA and a C genome specific DNA sequence in the genus Avena. Genome 39:535-542

Linares C, Ferrer E, Fominaya A (1998) Discrimination of the closely related $\mathrm{A}$ and $\mathrm{D}$ genomes of the hexaploid oat Avena sativa $\mathrm{L}$. Proc Natl Acad Sci 95:12450-12455

Loskutov IG (2001) Interspecific crosses in the genus Avena L. Russ J Genet 37:467-475

Loskutov IG (2008) On evolutionary pathways of Avena species. Genet Resour Crop Evol 55:211-220

Loskutov IG, Rines HW (2011) Avena. In: Kole C (ed) Wild crop relatives: genomic and breeding resources. Springer, Heidelberg, pp 109-183

Lu F, Lipka AE, Glaubitz J, Elshire R, Cherney JH, Casler MD, Buckler ES, Costich DE (2013) Switchgrass genomic diversity, ploidy, and evolution: novel insights from a network-based SNP discovery protocol. PLoS Genet 9(1):e1003215. doi:10.1371/ journal.pgen.1003215

Marshall HG, Myers WM (1961) A cytogenetic study of certain interspecific Avena hybrids and the inheritance of resistance in diploid and tetraploid varieties to races of crown rust. Crop Sci 1:29-34

Murphy HC, Sadanaga K, Zillinsky FJ, Terrell EE, Smith RT (1968) Avena magna: an important new tetraploid species of oats. Science 159:103-104

Nikoloudakis N, Katsiotis A (2008) The origin of the C-genome and cytoplasm of Avena polyploids. Theor Appl Genet 117:273-281

Nocelli E, Giovannini T, Bioni M, Alicchio R (1999) RFLP-and RAPD-based genetic relationships of seven diploid species of Avena with the A genome. Genome 42:950-959

Oliver RE, Jellen EN, Ladizinsky G, Korol AB, Kilian A, Beard JL, Dumlupinar Z, Wisniewski-Morehead NH, Svedin E, Coon M (2011a) New diversity arrays technology (DArT) markers for tetraploid oat (Avena magna Murphy et Terrell) provide the first complete oat linkage map and markers linked to domestication genes from hexaploid A. sativa L. Theor Appl Genet 123:1159-1171

Oliver RE, Lazo GR, Lutz JD, Rubenfield MJ, Tinker NA, Anderson JM, Morehead NHW, Adhikary D, Jellen EN, Maughan PJ (2011b) Model SNP development for complex genomes based on hexaploid oat using high-throughput 454 sequencing technology. BMC Genom 12:1

Oliver RE, Tinker NA, Lazo GR, Chao S, Jellen EN, Carson ML, Rines HW, Obert DE, Lutz JD, Shackelford I, Korol AB, Wight CP, Gardner KM, Hattori J, Beattie AD, Bjørnstad A, Bonman JM, Jannink J-L, Sorrells ME, Brown-Guedira GL, 
Mitchell Fetch JW, Harrison SA, Howarth CJ, Ibrahim A, Kolb FL, McMullen MS, Murphy JP, Ohm HW, Rossnagel BG, Yan W, Miclaus KJ, Hiller J, Maughan PJ, Redman Hulse RR, Anderson JM, Islamovic E, Jackson EW (2013) SNP discovery and chromosome anchoring provide the first physically-anchored hexaploid oat map and reveal synteny with model species. PLoS One 8:e58068

Peng Y-Y, Wei Y-M, Baum BR, Zheng Y-L (2008) Molecular diversity of the 5S rRNA gene and genomic relationships in the genus Avena (Poaceae: Aveneae). Genome 51:137-154

Peng Y-Y, Wei Y-M, Baum BR, Yan Z-H, Lan X-J, Dai S-F, Zheng Y-L (2010) Phylogenetic inferences in Avena based on analysis of $F L$ intron2 sequences. Theor Appl Genet 121:985-1000

Qi ZC, Yu Y, Liu X, Pais A, Ranney T, Whetten R, Xiang QYJ (2015) Phylogenomics of polyploid Fothergilla (Hamamelidaceae) by RAD-tag based GBS - insights into species origin and effects of software pipelines. J Syst Evol 53:432-447

R Core Team (2016) R: a language and environment for statistical computing. R Foundation for Statistical Computing, Vienna, Austria. http://www.R-project.org. (ISBN 3-900051-07-0)

Rajhathy T (1966) Evidence and an hypothesis for the origin of the C genome of hexaploid Avena. Can J Genet Cytol 8:774-779

Rajhathy T, Baum BR (1972) Avena damascena: a new diploid oat species. Can J Genet Cytol 14:645-654

Rajhathy T, Morrison JW (1960) Genome homology in the genus Avena. Can J Genet Cytol 2:278-285

Rajhathy T, Thomas H (1967) Chromosomal differentiation and speciation in diploid Avena. III. Mediterranean wild populations. Can J Genet Cytol 9:52-68

Rajhathy T, Thomas H (1974) Cytogenetics of oats (Avena L.) Miscellaneous Publications of the Genetics Society of Canada. Genetics Society of Canada, Ottawa

Rines HW, Porter HL, Carson ML, Ochocki GE (2007) Introgression of crown rust resistance from diploid oat Avena strigosa into hexaploid cultivated oat $A$. sativa by two methods: direct crosses and through an initial $2 \times \cdot 4 \times$ synthetic hexaploid. Euphytica 158:67-79

Sanz M, Jellen E, Loarce Y, Irigoyen M, Ferrer E, Fominaya A (2010) A new chromosome nomenclature system for oat (Avena sativa L. and A. byzantina C. Koch) based on FISH analysis of monosomic lines. Theor Appl Genet 121:1541-1552

Thomas H (1992) Cytogenetics of Avena. In: Marshall HG, Sorrells ME (eds) Oat science and technology. American Society of Agronomy, Crop Science Society of America, Madison, pp 473-507

Tinker NA, Chao S, Lazo GR, Oliver RE, Huang Y-F, Poland JA, Jellen EN, Maughan PJ, Kilian A, Jackson EW (2014) A SNP genotyping array for hexaploid oat. Plant Genome. doi:10.3835/ plantgenome2014.03.0010

Tinker NA, Bekele WA, Hattori J (2016) Haplotag: software for haplotype-based genotyping-by-sequencing analysis. G3 Genes Genomes Genet 6:857-863

Yan H-H, Baum BR, Zhou P-P, Zhao J, Wei Y-M, Ren C-Z, Xiong F-Q, Liu G, Zhong L, Zhao G, Peng Y-Y, Scoles G (2014) Phylogenetic analysis of the genus Avena based on chloroplast intergenic spacer $p s b \mathrm{~A}-t r n \mathrm{H}$ and single-copy nuclear gene Acc1. Genome 57:267-277

Yan H, Martin SL, Bekele WA, Latta RG, Diederichsen A, Peng Y, Tinker NA (2016) Genome size variation in the genus Avena. Genome 59:209-220

Yang Q, Hanson L, Bennett MD, Leitch IJ (1999) Genome structure and evolution in the allohexaploid weed Avena fatua L. (Poaceae). Genome 42:512-518

Zamir D (2001) Improving plant breeding with exotic genetic libraries. Nat Rev Genet 2:983-989 